\title{
Infralimbic Prefrontal Cortex Is Responsible for Inhibiting Cocaine Seeking in Extinguished Rats
}

\author{
Jamie Peters, Ryan T. LaLumiere, and Peter W. Kalivas \\ Department of Neurosciences, Medical University of South Carolina, Charleston, South Carolina 29425
}

The rat prelimbic prefrontal cortex and nucleus accumbens core are critical for initiating cocaine seeking. In contrast, the neural circuitry responsible for inhibiting cocaine seeking during extinction is unknown. The present findings using inhibition of selected brain nuclei with GABA agonists show that the suppression of cocaine seeking produced by previous extinction training required activity in the rat infralimbic cortex. Conversely, the reinstatement of drug seeking by a cocaine injection in extinguished animals was suppressed by increasing neuronal activity in infralimbic cortex with the glutamate agonist AMPA. The cocaine seeking induced by inactivating infralimbic cortex resembled other forms of reinstated drug seeking by depending on activity in prelimbic cortex and the basolateral amygdala. A primary efferent projection from the infralimbic cortex is to the nucleus accumbens shell. Akin to infralimbic cortex, inhibition of the accumbens shell induced cocaine seeking in extinguished rats. However, bilateral inhibition of the shell also elicited increased locomotor activity. Nonetheless, unilateral inhibition of the accumbens shell did not increase motor activity, and simultaneous unilateral inactivation of the infralimbic cortex and shell induced cocaine seeking, suggesting that an interaction between these two structures is necessary for extinction training to inhibit cocaine seeking. The infralimbic cortex and accumbens shell appear to be recruited by extinction learning because inactivation of these structures before extinction training did not alter cocaine seeking. Together, these findings suggest that a neuronal network involving the infralimbic cortex and accumbens shell is recruited by extinction training to suppress cocaine seeking.

Key words: infralimbic; prelimbic; nucleus accumbens; amygdala; cocaine seeking; extinction

\section{Introduction}

Exposure to drug-associated cues in cocaine addicts produces activation within the anterior cingulate cortex, including Brodmann's areas (BA) 24 and 32, which correlates with self-reports of cue-induced craving (Grant et al., 1996; Childress et al., 1999). Inhibition of the homologous prelimbic cortex in rats (Ongür and Price, 2000) blocks multiple forms of reinstated drug seeking, including cue-, drug-, and stress-induced cocaine seeking (McFarland and Kalivas, 2001; Capriles et al., 2003; McLaughlin and See, 2003; McFarland et al., 2004). These behavioral and imaging studies, together with numerous neurochemical, electrophysiological, and pharmacological data, strongly implicate activation of the dorsal prefrontal cortex in initiating cocaine seeking (Kalivas and O'Brien, 2008).

In addition to initiating learned behaviors such as drug seeking, the prefrontal cortex is thought to suppress the expression of learned motivated behaviors, and damage to regions of ventral

Received Nov. 28, 2007; revised May 3, 2008; accepted May 4, 2008.

This work was supported by National Institute on Drug Abuse Grants DA-12513 (P.W.K.), DA-18486 (J.P.), and DA-21460 (R.T.L.), as well as Program Project DA-05369. We thank Dr. Gregory Quirk (University of Puerto Rico, San Juan, Puerto Rico) and his laboratory and Dr. Abraham Zangen (Weizmann Institute of Science, Rehovot, Israel) for their helpful comments. Also, Dr. Rita Fuchs (University of North Carolina, Chapel Hill, NC) provided invaluable advice over the course of conducting these experiments.

Correspondence should be addressed to Jamie Peters at her present address: Department of Psychiatry, University of Puerto Rico School of Medicine, P0 Box 365067, San Juan, Puerto Rico 00936-5067. E-mail: petersjl.upr@ gmail.com.

D0I:10.1523/JNEUROSCI.1045-08.2008

Copyright $\odot 2008$ Society for Neuroscience $\quad 0270-6474 / 08 / 286046-08 \$ 15.00 / 0$ prefrontal cortex encompassing BA10-BA13 and BA25 have been associated with the loss of inhibitory control (Bechara, 2005). The homologous region in rat prefrontal cortex is the infralimbic cortex (Ongür and Price, 2000), and a series of studies show that activity in the infralimbic cortex is necessary for extinction training to suppress conditioned fear responses (Quirk et al., 2006). Moreover, it was shown recently that the prelimbic prefrontal cortex is necessary to initiate conditioned fear responses (Vidal-Gonzalez et al., 2006), suggesting a functional dichotomy within the prefrontal cortex in which the prelimbic cortex initiates a conditioned fear response and the infralimbic cortex is recruited by extinction training to suppress conditioned fear responding.

The present study was undertaken to determine whether, akin to fear conditioning, a functional dichotomy in prefrontal cortex exists for cocaine-seeking behavior. Because it is well established that activity in the prelimbic cortex initiates cocaine seeking, we hypothesized that activity in infralimbic cortex contributes to the inhibition of cocaine seeking produced by extinction training. Also, activity in the projection from the prelimbic cortex to the nucleus accumbens core (NAcore) is necessary to reinstate cocaine seeking (Kalivas and O'Brien, 2008). Because the infralimbic cortex has a parallel projection to the shell subcompartment of the accumbens (NAshell) (Sesack et al., 1989), we also hypothesized that the NAshell contributes to the suppression of behavior in extinguished animals. Finally, other brain regions projecting to the prelimbic cortex and NAcore have been implicated in initiat- 
ing cocaine seeking, including the basolateral amygdala (BLA) (Di Ciano and Everitt, 2004). Because the first experiment revealed that activity in the infralimbic cortex was necessary for suppressing cocaine seeking in extinguished rats, we hypothesized that the cocaine seeking elicited by inhibiting activity in infralimbic cortex would rely on an interconnected circuit containing the BLA and prelimbic cortex.

\section{Materials and Methods}

Animal housing and surgery. All procedures were in accordance with the National Institutes of Health Guide for the Care and Use of Laboratory Animals and the Assessment and Accreditation of Laboratory Animal Care. Male Sprague Dawley rats ( 243 total; 250 g on arrival; Charles River Laboratories) were individually housed in a temperature- and humiditycontrolled environment with a $12 \mathrm{~h}$ light/dark cycle (7:00 A.M. lights on). Experiments were conducted during the first $8 \mathrm{~h}$ of the rats' dark cycle. Rats received food ad libitum until $1 \mathrm{~d}$ before behavioral training, after which food restriction procedures ( $20 \mathrm{~g}$ of rat chow per day) were implemented and maintained throughout the duration of the experiment, and water was always available ad libitum in the home cage. Rats were allowed $\sim 1$ week to acclimate to the vivarium before inducing anesthesia and implanting jugular and intracranial cannula (for surgical details, see McFarland and Kalivas, 2001). Intracranial guide cannulas were stereotaxically implanted bilaterally into the infralimbic cortex, prelimbic cortex, NAcore, NAshell, or BLA, or into the infralimbic cortex and one of the other aforementioned structures. A diagram illustrating each experimental protocol is shown in supplemental Figure 1 (available at www. jneurosci.org as supplemental material).

Cocaine self-administration procedures. One week after surgery, food restriction procedures were implemented, and, for the duration of the experiment, rats received a ration of $20 \mathrm{~g}$ of rat chow per day. Rats were initially allowed to lever press for food for up to $15 \mathrm{~h}$ until they were shaped on the right lever. The following day, rats began cocaine selfadministration. During this phase, each response on the right lever resulted in an infusion of cocaine (National Institutes of Health, Bethesda, MD) dissolved in sterile $0.9 \%$ saline $(0.2 \mathrm{mg}$ in $0.05 \mathrm{ml}$ for $12 \mathrm{~d})$. A timeout period of $20 \mathrm{~s}$ followed each intravenous infusion, wherein responses on the right lever were without consequence, which was intended to help prevent overdose. No discrete cues were present during training. Failure to self-administer to a criterion of at least 15 infusions/d over the last $2 \mathrm{~d}$ of self-administration resulted in elimination from the study $(n=8)$.

Microinfusion procedure for extinction studies. The GABA agonists baclofen plus muscimol (Bac+Mus; $0.3+0.03 \mathrm{nmol} /$ side, respectively) were chosen to pharmacologically inactivate discrete, often adjacent brain regions, because they produce a rapid and prolonged reduction in neuronal activity without inhibiting fibers of passage (Martin and Ghez, 1999; van Duuren et al., 2007). Immediately before placement in the operant chamber on the test sessions, rats were microinjected with either PBS or Bac+Mus into each hemisphere simultaneously in a betweensubjects manner. All microinjections were made in a volume of $0.3 \mu \mathrm{l} /$ side over $1 \mathrm{~min}$. After allowing $1.5 \mathrm{~min}$ for diffusion, the microinjectors were removed, and animals were then placed in the operant box for $2 \mathrm{~h}$. The animals in the PBS and Bac+Mus groups were assigned after training to self-administer cocaine to ensure that the two microinjection groups had equivalent levels of active lever pressing on the last $2 \mathrm{~d}$ of self-administration training.

Microinjections during extinction training. Behavioral testing was conducted after microinjecting PBS or Bac+Mus on the first day extinction training or after $12 \mathrm{~d}$ of training. Each brain structure was examined on the first day of extinction training to assess its role in cocaine seeking before any extinction learning and on day 12 of training to assess the role of each structure in the expression of extinction after extinction was well learned. For day 1 testing, average responding over the last $2 \mathrm{~d}$ of selfadministration and responding on the drug-free extinction session of the following day (day 2) were also analyzed. For day 12 testing, both a pretest and posttest baseline extinction level of responding was measured by averaging the number of lever presses during the two extinction ses- sions before and after the microinjeciton of PBS or Bac+Mus. If the pretest baseline was $>40$ presses, the subject was not tested further $(n=$ 16). Because no differences were observed between the pretest and posttest extinction baselines, these values were averaged to obtain a single extinction value for each subject in the data presentation.

Disconnection study. Extinction testing was conducted during extinction days $10-16$, because each subject received two tests separated by at least three extinction sessions. A single extinction baseline value for each subject was obtained by averaging responding during the two extinction sessions before and after the microinjection test, because these pretest and posttest baselines did not differ. For this study, rats were bilaterally cannulated over both infralimbic cortex and NAshell. There were four sets of microinjections, with PBS versus Bac + Mus in each set: unilateral infralimbic, unilateral NAshell, unilateral ipsilateral infralimbic-NAshell (each structure is microinjected unilaterally in the same hemisphere), and contralateral infralimbic-NAshell (each structure is microinjected unilaterally in opposite hemispheres). Because the four PBS groups did not differ in their extinction baseline or responding after microinjection, these groups were pooled to form the single PBS group used for statistical analysis. Treatment groups were pseudorandomized such that each subject received a microinjection through each cannulas no more than once. When more than one unilateral microinjection was used, rats received both microinjections simultaneously. All other microinjection parameters were identical to those described for extinction testing.

Infralimbic reinstatement study. Testing was conducted on extinction day 12 in a between-subjects manner to determine whether inhibition of the prelimbic cortex or BLA could suppress the capacity of infralimbic inactivation to induce cocaine seeking. A single extinction baseline value for each subject was obtained by averaging responding during the two extinction sessions before and after the microinjection day, because these pretest and posttest baselines did not differ. Rats were bilaterally cannulated over both infralimbic cortex and prelimbic cortex or over both infralimbic cortex and BLA. Each rat received infralimbic Bac+Mus combined with microinfusion of either Bac+Mus or PBS into the prelimbic cortex or the BLA. All other microinjection parameters were identical to those described above, and each animal was tested only once.

AMPA study. The AMPA study was conducted in a within-subjects manner, such that each rat received a microinjection of PBS and AMPA bilaterally into infralimbic cortex in a randomized order on different test days, separated by at least $3 \mathrm{~d}$ of extinction. AMPA was microinjected at a dose of $0.1 \mathrm{nmol} / \mathrm{side}$, in a $0.3 \mu \mathrm{l}$ volume over $1 \mathrm{~min}$. After allowing 1.5 min for diffusion, the microinjectors were removed, and a priming injection of cocaine $(10 \mathrm{mg} / \mathrm{kg}$, i.p.) was administered to the rat just before its placement in the operant chamber for testing. Three rats did not exhibit significant cocaine-induced reinstatement with PBS $(<40$ presses) and were eliminated from the study. As a control, a small number of animals were tested on day 12 of extinction session in the absence of a cocaine priming injection to verify that this dose of AMPA produced no significant effects on extinction responding.

Locomotor study. A subset of rats used for infralimbic cortex and NAshell extinction studies were tested for locomotor effects of bilateral inactivation with Bac+Mus. Behavioral chambers contained 16 photobeams (AccuScan Instruments), and each rat was microinjected immediately before placement in the locomotor chamber for a $2 \mathrm{~h}$ locomotor test session.

Histology and statistics. After experimentation, rats were administered pentobarbital ( $\sim 350 \mathrm{mg} / \mathrm{kg}$, i.p.) and perfused transcardially with $10 \%$ Formalin. Coronal sections $(150 \mu \mathrm{m})$ were made through the cannula tract and stained with cresyl violet to verify anatomical placement $(n=$ 26 eliminated) according to the atlas of Paxinos and Watson (1986). Two-way repeated-measures ANOVAs were used to determine effects on cocaine seeking (GraphPad/Prism software). A Bonferroni's post hoc analysis was conducted to determine whether there was a significant difference between groups. For the locomotor activity study, a two-tailed Student's $t$ test was used to determine effects of drug on total distance traveled. Statistical analyses are described in detail in each figure legend. 


\section{Results}

Inhibition of the infralimbic cortex

induces cocaine seeking in

extinguished animals

Rats were trained to self-administer cocaine, divided into PBS or Bac+Mus groups, and placed in extinction training. Figure $1 A$ shows that both groups had equivalent levels of cocaine selfadministration over the last $2 \mathrm{~d}$ of training and significantly distinguished between the active and inactive levers. On day 12 of extinction, microinjections of PBS or $\mathrm{Bac}+$ Mus were administered into the infralimbic cortex before placing the rat into the operant chamber. Extinction was well learned by the time of testing, indicated by the low extinction baseline in each group, as well as the low number of active lever presses in animals microinjected with PBS. Bilateral inactivation of the infralimbic cortex (Fig. 1A) with Bac+Mus reinstated lever pressing relative to the PBS controls on the active lever. Inhibition of the infralimbic cortex also increased inactive lever pressing, although the increase in active lever pressing was significantly greater than inactive lever pressing (Fig. $1 A$ ). In considering the increase in inactive lever pressing, it is important to note that, in the absence of extinction training, such as on the first day of extinction training, inactive lever pressing is also increased to a similar extent (Fig. 1A) (see Fig. 5) and, in this case, reflects alternate drug seeking rather than nonspecific behavioral activation.

Nonetheless, because inactive lever was elevated, locomotor testing was conducted to determine the effects of infralimbic cortex on motor activity. Inactivation of infralimbic cortex produced no effect on locomotion (mean \pm SEM centimeters traveled over 120 min after injection: PBS, 11,462 $\pm 375, n=10$; Bac+Mus, $15,767 \pm 2367, n=6)$.

\section{Activation of infralimbic cortex inhibits cocaine-induced reinstatement}

If activity in infralimbic cortex is necessary for the behavioral inhibition learned during extinction training, stimulating infralimbic cortex should resemble activation of an extinction memory and suppress cocaine seeking. Neurons in the infralimbic cortex were activated by microinjecting the glutamate agonist AMPA immediately before reinstating cocaine seeking with a noncontigent injection of cocaine $(10 \mathrm{mg} / \mathrm{kg}$, i.p.). Figure $1 B$ shows that AMPA microinjection into infralimbic cortex reduced drug seeking induced by cocaine. AMPA alone in the infralimbic cortex failed to show reinstatement compared with the PBS controls.

\section{Inactivation of the NAshell induces cocaine seeking in extinguished rats}

A major efferent projection from the infralimbic cortex is to the NAshell (Sesack et al., 1989), and a number of pharmacological studies show that the NAshell can both promote and inhibit motivated behaviors (Di Chiara, 2002; Kelley, 2004). Figure $2 \mathrm{~A}$ shows that, akin to the infralimbic cortex, inactivation of the NAshell after $11 \mathrm{~d}$ of extinction training elicited an increase in both active and inactive lever pressing, and the increase in active lever pressing was greater than inactive lever responding. However, bilateral, but not unilateral, inactivation of the NAshell elicited a significant increase in motor activity (Fig. $2 \mathrm{~B}$ ). Thus, in contrast to the infralimbic cortex, nonspecific motor activation may contribute to the increase in cocaine seeking after NAshell inactivation.

To assess a shared involvement of the infralimbic cortex and NAshell in suppressing cocaine seeking, rats were implanted with cannulas over both the infralimbic cortex and NAshell, and $\mathrm{Bac}+\mathrm{Mus}$ was administered unilaterally into each region in a contralateral or ipsilateral manner. Because the infralimbic to NAshell projection is substantially bilateral (Sesack et al., 1989), this technique cannot be used to validate a role of these brain regions as a series projection regulating cocaine seeking (McFarland and Kalivas, 2001; Di Ciano and Everitt, 2004). Nonetheless, disconnection of a bilateral pathway can establish that the two structures participate in a network if the effects of simultaneous unilateral inactivation of both structures is greater than the additive effect of unilateral inactivation in each structure alone. Figure $2 C$ shows that unilateral administration of $\mathrm{Bac}+\mathrm{Mus}$ into either the infralimbic cortex or NAshell did not reinstate lever pressing. When the infralimbic cortex and NAshell were simultaneously inactivated in either contralateral or ipsilateral hemispheres, active and inactive lever responding was in- 

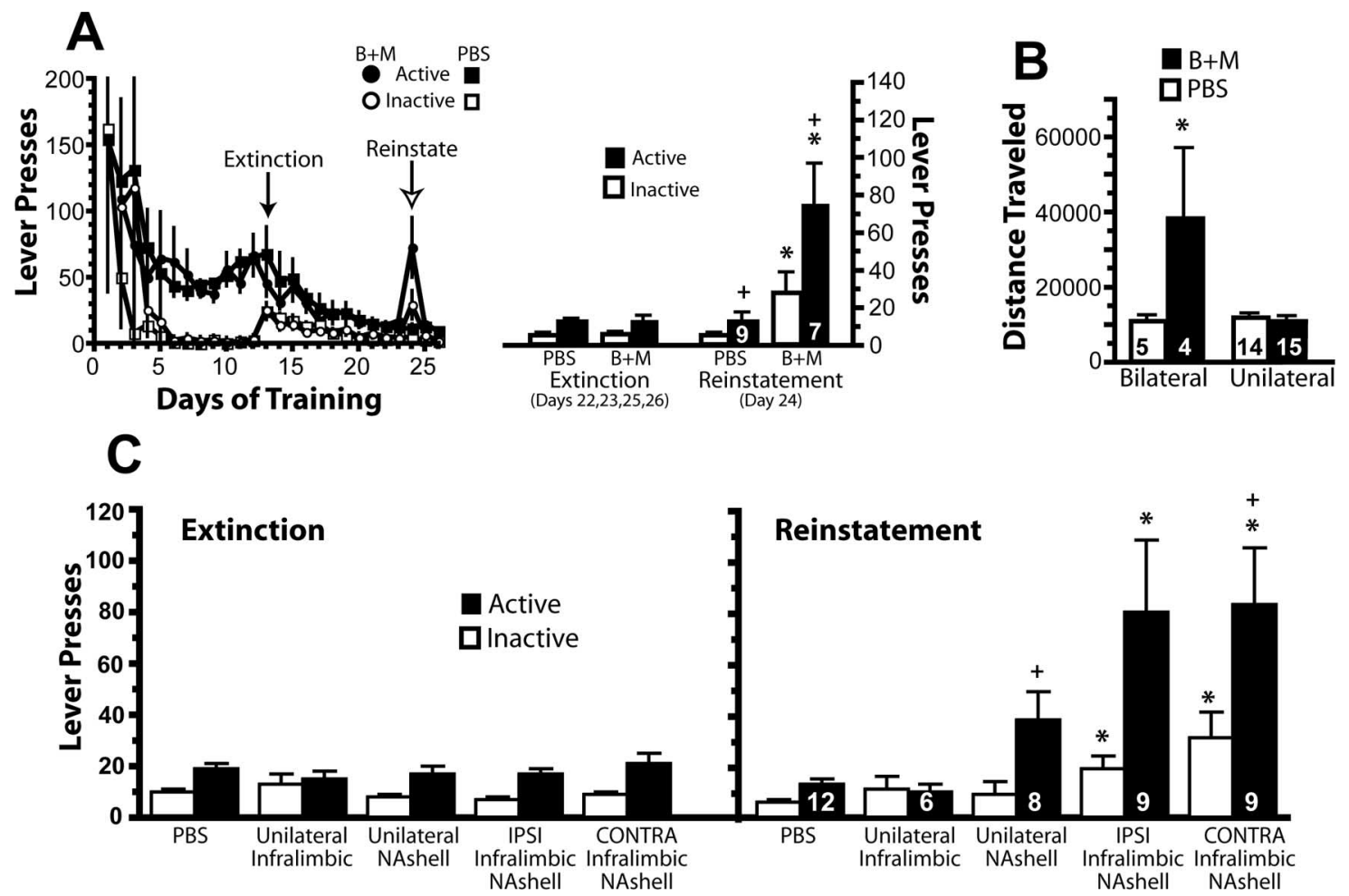

Figure 2. Inactivation of the NAshell induces reinstatement after $11 \mathrm{~d}$ of extinction training, and disconnection of the infralimbic cortex and NAshell also reinstates cocaine seeking. $\boldsymbol{A}$, The left shows the active and inactive lever pressing on each day of the experiment, and the right shows the average extinction baseline pressing and pressing after microinjecting PBS control or baclofen plus muscimol $(B+M)$ into the infralimbic cortex. Data were analyzed using a two-way ANOVA with repeated measures over lever, and, for the reinstatement day, a significant effect of treatment $\left(F_{(1,14)}=9.27, p=0.009\right)$, lever $\left(F_{(1,14)}=13.82, p=0.002\right)$, and interaction $\left(F_{(1,14)}=6.69, p=0.022\right)$ was measured. $\boldsymbol{B}$, Bilateral inactivation of NAshell induced increased locomotor activity (Student's $t$ test, $t_{(10)}=4.29, p=0.002$ ), whereas unilateral inactivation did not. $C$, The left shows the active and inactive lever pressing during the extinction baseline sessions. In the right, microinjections of PBS or Bac + Mus were made unilaterally into the infralimbic cortex alone (Unilateral Infralimbic), the NAshell alone (Unilateral NAshell), or into both structures simultaneously, in either an ipsilateral (IPSI Infralimbic NAshell) or contralateral (CONTRA Infralimbic NAshell) manner just before the reinstatement test session. PBS groups were pooled to obtain the single PBS group shown here. Other groups indicate Bac + Mus injections. Data were analyzed using a two-way ANOVA, and, for the reinstatement day, a significant effect of treatment $\left(F_{(4,78)}=5.79, p<\right.$ $0.001)$ and lever $\left(F_{(1,78)}=12.99, p=0.001\right)$, but no interaction, was measured. Numbers in bars indicate $n .{ }^{*} p<0.05$ compared with respective PBS group using a Bonferroni's post hoc test. ${ }^{+} p<$ 0.05 compared with inactive lever on the reinstatement test using a Bonferroni's post hoc test.

creased relative to the PBS controls and to the extinction baseline. As with drug seeking after bilateral inactivation of either the infralimbic cortex (Fig. 1A) or NAshell (Fig. 2A), the increase in active lever pressing after simultaneous contralateral inactivation was greater than the increase in inactive lever responding.

A BLA-prelimbic circuit is required for cocaine seeking elicited by inhibiting infralimbic cortex

Inactivation of the prelimbic cortex (Fig. 3A) or the BLA (Fig. 3B) did not alter active or inactive lever pressing in animals extinguished from cocaine self-administration. In contrast, inactivation of the NAcore caused a significant decrease in active lever pressing in the Bac+Mus group compared with PBS controls (Fig. 3C). Extinction baselines were not different between treatment groups.

The reinstatement of cocaine seeking requires activity in the prelimbic prefrontal cortex, and, in the case of cue-induced reinstatement, the BLA is also required (McLaughlin and See, 2003; Di Ciano and Everitt, 2004). To determine whether cocaine seeking induced by infralimbic cortex inactivation resembles other forms of reinstated drug seeking, rats were administered $\mathrm{Bac}+\mathrm{Mus}$ or PBS into either the prelimbic cortex or the BLA immediately before inactivating infralimbic cortex. As with cueinduced reinstatement of cocaine seeking, infralimbic inactivation-induced cocaine seeking was found to rely on activity in both the prelimbic cortex and BLA (Fig. 3D). Thus,
$\mathrm{Bac}+$ Mus into either the prelimbic cortex or BLA before infralimbic inactivation reduced cocaine seeking compared with infralimbic inactivation alone. In this set of experiments, the increase in active lever pressing by inactivating infralimbic cortex alone was not associated with increased inactive lever pressing (Fig. 3D).

The infralimbic and prelimbic cortex do not regulate cocaine seeking on the first day of extinction

To determine whether the role of the prefrontal cortex in cocaine seeking results from adaptations produced by cocaine selfadministration or by extinction training, the effects of infralimbic or prelimbic inactivation on cocaine seeking during the first day of extinction were examined. Figure $4, A$ and $B$, shows that inactivation of either region of the prefrontal cortex did not alter active or inactive lever pressing on the first day of extinction training compared with rats injected with PBS into the infralimbic cortex. For both the infralimbic and prelimbic cortex, inactive lever pressing was increased on the first day of extinction relative to the self-administration baseline, regardless of whether the animals were injected with PBS or Bac+Mus, consistent with animals engaging in alternate drug seeking when the active lever failed to deliver cocaine.

The NAcore and NAshell were also inactivated by Bac + Mus on the first day of extinction. Inactivation of the NAshell did not alter active or inactive lever pressing relative to PBS injection (Fig. 
4C). However, inactivation of the NAcore reduced active lever pressing (Fig. $4 D$ ). In addition, on the following drug-free extinction session, animals that had received $\mathrm{Bac}+$ Mus inactivation of the NAcore on day 1 exhibited a rebound of responding on day 2 similar to day 1 response rates in the PBS controls, suggesting that they failed to learn extinction under the influence of Bac+Mus on day 1 .

The BLA was also inactivated on the first day of extinction, and there is some evidence for an inhibitory effect of inactivation in that PBS control animals showed a significant reduction in active lever pressing between days 1 and 2 of extinction and $\mathrm{Bac}+$ Mus animals did not, primarily because of a near-significant inhibition of lever pressing on day 1 . However, future studies are necessary to determine involvement of the BLA in regulating extinction learning. Importantly, for each brain area, animals were separated into the PBS and Bac+Mus groups such that the two treatment groups had equivalent levels of active and inactive lever pressing during the last $2 \mathrm{~d}$ of cocaine selfadministration (Fig. 4).

\section{Histology}

An illustration of all microinjection sites is shown in supplemental Figure 2 (available at www.jneurosci.org as supplemental material). Infralimbic microinjections were made within the boundaries of this area of the prefrontal cortex. Prelimbic microinjections included the prelimbic proper and aspects of anterior cingulate. NAcore microinjections were located within $0.5 \mathrm{~mm}$ of the anterior commissure, and microinjections into the NAshell were focused in the ventromedial portion of the nucleus. BLA infusions were primarily located in the caudal basal nucleus but also portions of the lateral nucleus.

\section{Discussion}

The present study demonstrates that neural activity within the infralimbic cortex is necessary for the suppression of cocaine seeking attributable to extinction training. Thus, infralimbic cortex inactivation by GABA agonists reinstated cocaine seeking, and increasing neuronal activity with AMPA inhibited cocaine-induced reinstatement. The infralimbic cortex has a dense glutamatergic projection to the NAshell (Sesack et al., 1989), and both bilateral inactivation of the NAshell or simultaneous unilateral inactivation of the infralimbic cortex and the NAshell induced cocaine seeking. These data support the infralimbic cortex and NAshell as participants in a functional network that mediates extinction-induced inhibition of cocaine

D
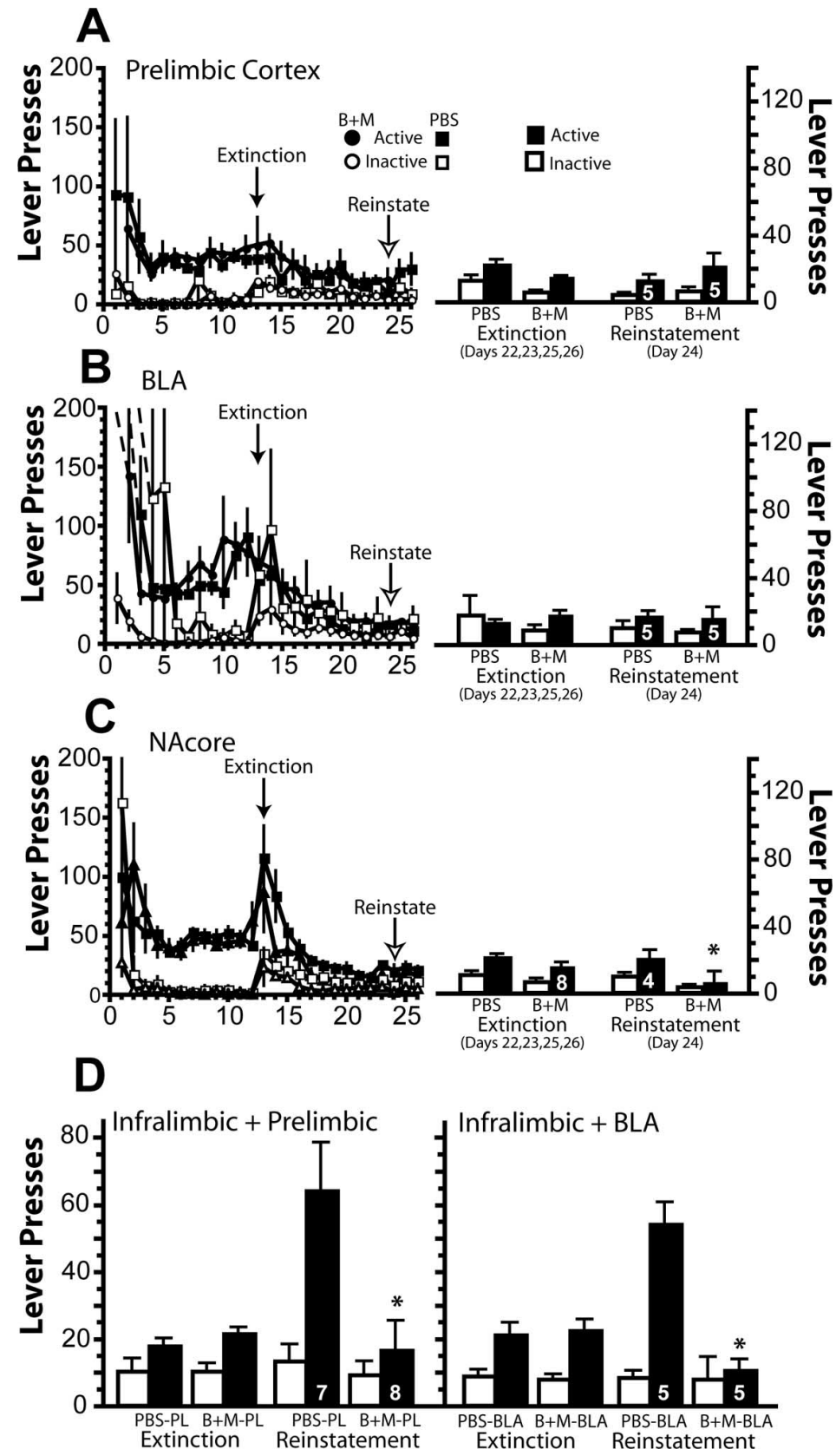

Figure 3. Inactivation of prelimbic cortex, BLA, or NAcore did not reinstate cocaine seeking after 11 d of extinction training, but inactivation of prelimbic cortex and BLA prevented reinstatement induced by inactivating the infralimbic cortex. The left shows the active and inactive lever pressing on each day of the experiment, and the right shows the average extinction baseline pressing and pressing after microinjecting PBS control or baclofen plus muscimol $(B+M)$ into each structure shown in $A-C$. $A$, A two-way ANOVA of prelimbic data showed only a significant effect of lever $\left(F_{(1,8)}=5.14, p=0.053\right)$. $\boldsymbol{B}$, A two-way ANOVA of BLA data showed no significant effects. $C$, A two-way ANOVA of NAcore data showed significant effects of treatment $\left(F_{(1,14)}=6.67, p=\right.$ $0.021)$ and lever $\left(F_{(1,14)}=4.69, p=0.048\right)$ but no interaction. $D$, The left shows the average extinction baseline pressing and pressing after microinjecting PBS control or baclofen plus muscimol $(B+M)$ into the prelimbic cortex, and the right into $B L A$, in conjunction with a microinjection of $B+M$ into infralimbic cortex. A two-way ANOVA of the dual inactivation of the infralimbic cortex and prelimbic cortex show significant effects of treatment $\left(F_{(1,26)}=6.86, p=0.015\right)$, lever $\left(F_{(1,16)}=10.63, p=0.003\right)$, and a significant interaction $\left(F_{(1,26)}=4.34, p=0.047\right)$. A two-way ANOVA of the dual inactivation of the infralimbic cortex and BLA show significant effects of treatment $\left(F_{(1,16)}=13.67, p=0.002\right)$, lever $\left(F_{(1,16)}=17.86, p<0.001\right)$, and a significant interaction $\left(F_{(1,16)}=15.01, p=0.001\right)$. Numbers in bars indicate $n$. ${ }^{*} p<0.05$ comparing PBS and $B+M(A-C)$ or inactivation of both brain regions versus infralimbic inactivation alone $(\boldsymbol{D})$.

seeking. Although this finding is consistent with a previous finding that NMDA blockade in the NAshell reinstates cocaine seeking (Famous et al., 2007), bilateral NAshell inactivation elicited motor activity, as reported previously (Maldonado-Irizarry and Kelley, 1994; Kelley, 2004). This poses the possibility that the 

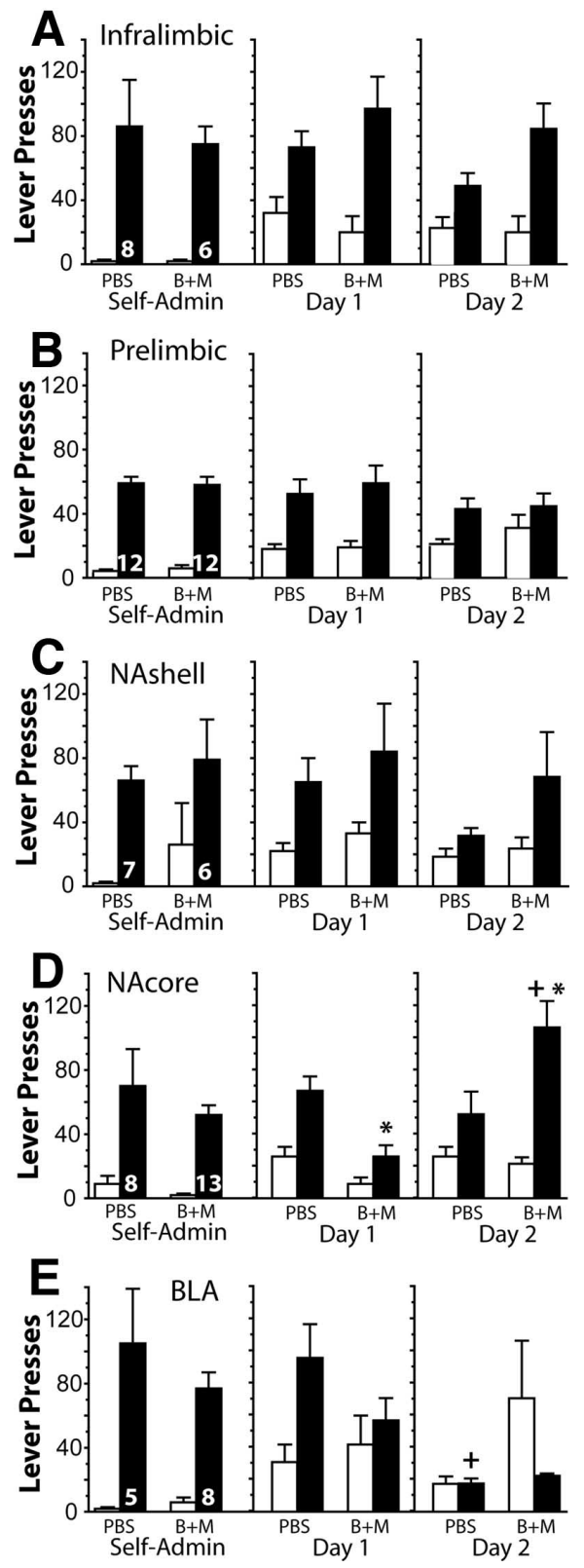

Figure 4. Prefrontal cortex regulation of cocaine seeking is recruited by extinction training. Animals were microinjected with PBS or Bac + Mus on the first day of extinction training (extinction day 1) before being placed in the behavioral chamber. Active and inactive lever pressing is shown for baseline pressing during the self-administration (Self-Admin) phase of the experiment, the extinction day 1 test (Day 1), and the following drug-free extinction session (Day 2). $A$, A two-way ANOVA of infralimbic data showed only a significant effect of lever $\left(F_{(1,48)}=\right.$ $43.62, p<0.001) . B, A$ two-way ANOVA of prelimbic data showed only a significant effect of lever $\left(F_{(1,104)}=29.64, p<0.001\right)$. C, A two-way ANOVA of NAshell data showed only a significant effect of lever $\left(F_{(1.44)}=12.82, p=0.001\right)$. $\boldsymbol{D}$, A two-way ANOVA of NAcore data showed a significant effect of treatment $\left(F_{(3,76)}=10.72, p<0.001\right)$, lever $\left(F_{(1,76)}=34.67\right.$, $p<0.001)$, and interaction $\left(F_{(3,76)}=4.91, p=0.004\right) . E$, A two-way ANOVA of BLA data showed significant interaction $\left(F_{(3,44)}=3.02, p=0.040\right)$ but no effect of treatment or lever. Numbers in bars indicate $n .{ }^{*} p<0.05$ comparing Bac + Mus with PBS on each day using a Bonferroni's post hoc test. ${ }^{+} p<0.05$ comparing days of extinction within each treatment using a Bonferroni's post hoc test.

NAshell may be less specifically engaged by extinction training and may serve a more general role in behavioral inhibition. Indeed, the fact that inhibition of dopamine transmission in the NAshell prevents cocaine-induced reinstatement indicates that the role of the NAshell in regulating cocaine seeking may vary

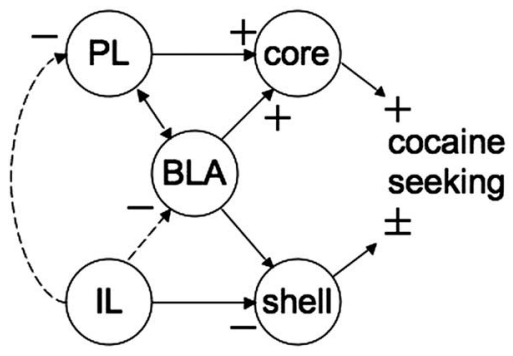

Figure 5. Hypothetical circuit whereby infralimbic cortex exerts inhibitory control over cocaine seeking. Glutamatergic projections from the prelimbic cortex (PL) and the basolateral amygdala (BLA) to the nucleus accumbens core (core) are known to drive cocaine-seeking behavior. Infralimbic cortex (IL) is proposed to indirectly inhibit both of these outputs to the core. In addition, the infralimbic projection to the NAshell (shell) exerts inhibitory regulation over cocaine seeking. It remains to be determined whether the projection from the BLA to the shell also exerts inhibitory control over cocaine seeking or whether the BLA and infralimbic glutamatergic afferents provide behaviorally competitive regulation of NAshell output. Bidirectional arrows indicate reciprocal projections between nuclei, and unidirectional indicates that the projection is rectified in the indicated direction. Solid lines indicate a direct projection. Dashed lines indicate that the projection may be indirect. + indicates output that promotes cocaine seeking. - indicates output that inhibits cocaine seeking. \pm indicates that the shell output can bidirectionally regulate cocaine seeking.

depending on experimental circumstances (Anderson et al., 2006; Schmidt et al., 2006). Moreover, the NAshell regulates different motivated behaviors, such as fear and feeding, along a rostrocaudal gradient, indicating that distinct neuronal ensembles in the NAshell may subserve modulatory roles in regulating distinct behaviors (Reynolds and Berridge, 2003).

\section{Prelimbic and infralimbic cortex in extinction and cocaine seeking}

In contrast to the inhibition of cocaine seeking associated with infralimbic activation, potentially via its projections to the NAshell, activation of prelimbic cortex and its projections to the NAcore are well characterized in promoting cocaine seeking (Kalivas and O'Brien, 2008). Thus, akin to previous work investigating fear conditioning (Quirk et al., 2006), in animals extinguished from cocaine self-administration, activity in the prelimbic cortex initiates behavioral responding (Kalivas and O'Brien, 2008), whereas in the present study, activity in infralimbic cortex inhibited behavioral responses. That these prefrontal subdivisions bidirectionally modulate both fear and cocaine seeking suggests they do not play a direct role in the motoric component of the behavioral response, because fear is assessed as motor inhibition (freezing), whereas cocaine seeking is measured as a facilitated motor output (increased lever pressing). Rather, the prelimbic and infralimbic cortices likely regulate the decision to engage or suppress conditioned responses. For conditioned fear, the infralimbic cortex suppresses freezing by inhibiting output from the central nucleus of the amygdala (Quirk et al., 2003; Berretta et al., 2005) that controls brainstem structures involved in autonomic fear responses (Rizvi et al., 1991). For cocaine seeking, infralimbic output directly or indirectly influences the NAshell, prelimbic cortex, and BLA to inhibit lever pressing (Fig. 5).

Importantly, obligatory involvement of both prefrontal regions in regulating cocaine seeking appears to require extinction training in this animal model of relapse. The role of infralimbic cortex was anticipated because previous studies showed that extinction training recruits infralimbic cortex to inhibit conditioned fear responding (Quirk et al., 2006). The shared infralimbic regulation of emotional behaviors lends support to previous 
suggestions that the homologous cortex in humans may be involved in voluntary suppression of emotional memories (Depue et al., 2007). Although most addicts do not undergo explicit extinction training, addiction is defined in part by an inability to cease drug taking even when a desire to suppress drug taking is declared. Hence, it is reasonable that the neural circuitry involved in actuating the desire to suppress drug seeking in humans may share circuits involved in expressing extinction in rats.

The inability of prelimbic inactivation to inhibit cocaine seeking on the first day of extinction was surprising because similar inactivation inhibits cocaine-, cue-, and stress-induced reinstatement of cocaine seeking in extinguished animals (McFarland and Kalivas, 2001; Capriles et al., 2003; Di Ciano and Everitt, 2004; McFarland et al., 2004). However, prelimbic cortex inactivation does not inhibit cocaine seeking in animals withdrawn from selfadministration that do not undergo extinction training (Fuchs et al., 2006). Rather, in this no-extinction model, only inactivation of the dorsolateral caudate-putamen inhibited cocaine seeking. Because the dorsolateral striatum receives no prefrontal input (Voorn et al., 2004), obligatory involvement of prefrontal-accumbens glutamate projections in cocaine seeking may require extinction training, although this notion bears more detailed examination in future studies. Interestingly, however, if prefrontal cortex is electrically stimulated during abstinence, cocaine seeking is reduced in the first extinction session (Levy et al., 2007), suggesting that electrical stimulation of prefrontal cortex may mimic extinction training and/or that extinction training enhances activity in prefrontal cortex.

\section{Accumbens subcompartments in extinction training}

The respective accumbens subcompartments receiving prelimbic and infralimbic input appear to be differentially recruited by extinction training. Inactivation of the NAcore suppressed cocaine seeking on the first day of extinction and appeared to inhibit the formation of extinction memory. NAshell inactivation in contrast did not alter responding in the first extinction session. Potential recruitment of glutamatergic efferents to the NAshell by extinction training has been indicated by previous work showing that extinction training increases the glutamate receptor 1 subunit of the AMPA receptor within the shell and that these protein levels correlate with the degree of extinction and inversely correlate with the degree of cue-induced reinstatement of cocaine seeking (Sutton et al., 2003). Distinctions between NAshell and NAcore in other forms of excitatory neuroplasticity have also been identified by Martin et al. (2006), who showed an enduring loss of long-term depression in the NAcore but not the NAshell after withdrawal from cocaine self-administration.

\section{Circuitry model for prefrontal inhibition and initiation of cocaine seeking}

Although the prelimbic and infralimbic cortices appear to serve distinct functions in regulating cocaine seeking, they are part of an integrated neural network that determines the intensity of behavioral responding. Thus, inhibiting either the prelimbic cortex or BLA prevented cocaine seeking elicited by inactivating infralimbic cortex. Because activity in both the prelimbic cortex and BLA is required for cocaine seeking induced by conditioned cues (Kantak et al., 2002; McLaughlin and See, 2003; Di Ciano and Everitt, 2004), one interpretation is that these structures are directly or indirectly receiving infralimbic input and that, in the absence of such input, they initiate cocaine seeking (for examples of direct and indirect connections, see Berretta et al., 2005; Jones et al., 2005; Marowsky et al., 2005). However, it is also possible that all three regions project in parallel to a brain region responsible for integrating the three inputs. A model is proposed in Figure 5 that attempts to integrate the current findings with previous studies. Figure 5 illustrates the hypothesis that efferent projections from infralimbic cortex are capable of directly or indirectly regulating cocaine seeking by dampening output from the brain regions known to initiate cocaine seeking, including the prelimbic cortex (McFarland et al., 2003) and BLA efferents to the NAcore (Di Ciano and Everitt, 2004). The infralimbic cortex projection to the NAshell is hypothesized to contribute to the inhibitory regulation of cocaine seeking, but the NAshell is also directly regulated by structures that trigger cocaine seeking, such as the BLA. Thus, glutamate transmission in the NAshell may either inhibit or facilitate cocaine seeking depending on whether it is derived from infralimbic or BLA afferents, respectively, perhaps accounting for why studies have identified both facilitatory and inhibitory roles for the NAshell in drug seeking and other motivated behaviors (Kelley, 2004; Reynolds and Berridge, 2003; Anderson et al., 2006; Famous et al., 2007).

\section{Conclusions}

The present study demonstrates that activity in the infralimbic cortex is required for the inhibition of cocaine seeking in extinguished rats and thereby shows a functional dichotomy between the prelimbic and infralimbic cortex. The respective initiation and inhibition of cocaine seeking by prelimbic and infralimbic cortex was not present on the first day of extinction, indicating that both prefrontal areas are recruited by extinction training to regulate cocaine seeking. This is consistent with the notion that extinction training recruits prefrontal cortex to regulate the welllearned habit of pressing the active lever for cocaine that was acquired during self-administration training. Moreover, it can be hypothesized that the recruited prefrontal cortex is permitting the animal greater range of cocaine-seeking behavior, including inhibition if no cocaine is delivered on lever pressing, or reinstating cocaine seeking if a cue, cocaine injection, or stressor is experienced. Given the many studies indicating that hypofrontality and corresponding cognitive deficits in cocaine addicts contributes to compulsive drug seeking (Jentsch and Taylor, 1999; Goldstein and Volkow, 2002; Bechara, 2005), understanding the mechanisms whereby extinction training recruits prefrontal cortex to regulate cocaine seeking may provide new pharmacotherapeutic targets for treating addiction.

\section{References}

Anderson SM, Schmidt HD, Pierce RC (2006) Administration of the D2 dopamine receptor antagonist sulpiride into the shell, but not the core, of the nucleus accumbens attenuates cocaine priming-induced reinstatement of drug seeking. Neuropsychopharmacology 31:1452-1461.

Bechara A (2005) Decision making, impulse control and loss of willpower to resist drugs: a neurocognitive perspective. Nat Neurosci 8:1458-1463.

Berretta S, Pantazopoulos H, Caldera M, Pantazopoulos P, Paré D (2005) Infralimbic cortex activation increases c-Fos expression in intercalated neurons of the amygdala. Neuroscience 132:943-953.

Capriles N, Rodaros D, Sorge RE, Stewart J (2003) A role for the prefrontal cortex in stress- and cocaine-induced reinstatement of cocaine seeking in rats. Psychopharmacology (Berl) 168:66-74.

Childress AR, Mozley PD, McElgin W, Fitzgerald J, Reivich M, O’Brien CP (1999) Limbic activation during cue-induced cocaine craving. Am J Psychiatry 156:11-18.

Depue BE, Curran T, Banich MT (2007) Prefrontal regions orchestrate suppression of emotional memories via a two-phase process. Science 317:215-219.

Di Chiara G (2002) Nucleus accumbens shell and core dopamine: differential role in behavior and addiction. Behav Brain Res 137:75-114.

Di Ciano P, Everitt BJ (2004) Direct interactions between the basolateral 
amygdala and nucleus accumbens core underlie cocaine seeking behavior by rats. J Neurosci 24:7167-7173.

Famous KR, Schmidt HD, Pierce RC (2007) When administered into the nucleus accumbens core or shell, the NMDA receptor antagonist AP-5 reinstates cocaine-seeking behavior in the rat. Neurosci Lett 420:169-173.

Fuchs RA, Branham RK, See RE (2006) Different neural substrates mediate cocaine seeking after abstinence versus extinction training: a critical role for the dorsolateral caudate-putamen. J Neurosci 26:3581-3588.

Goldstein RA, Volkow ND (2002) Drug addiction and its underlying neurobiological basis: neuroimaging evidence for the involvement of the frontal cortex. Am J Psychiatry 159:1642-1652.

Grant S, London ED, Newlin DB, Villemagne VL, Liu X, Contoreggi C, Phillips RL, Kimes AS, Margolin A (1996) Activation of memory circuits during cue-elicited cocaine craving. Proc Natl Acad Sci USA 93:12040-12045.

Jentsch JD, Taylor JR (1999) Impulsivity resulting from frontostriatal dysfunction in drug abuse: implications for the control of behavior by reward-related stimuli. Psychopharmacology 146:373-390.

Jones BF, Groenewegen HJ, Witter MP (2005) Intrinsic connections of the cingulate cortex in the rat suggest the existence of multiple functionally segregated networks. Neuroscience 133:193-207.

Kalivas PW, O'Brien C (2008) Drug addiction as a pathology of staged neuroplasticity. Neuropsychopharm Rev 33:166-180.

Kantak KM, Black Y, Valencia E, Green-Jordan K, Eichenbaum HB (2002) Dissociable effects of lidocaine inactivation of the rostral and caudal basolateral amygdala on the maintenance and reinstatement of cocaineseeking behavior in rats. J Neurosci 22:1126-1136.

Kelley AE (2004) Ventral striatal control of appetitive motivation: role in ingestive behavior and reward-related learning. Neurosci Biobehav Rev 27:765-776.

Levy D, Shabat-Simon M, Shalev U, Barnea-Ygael N, Cooper A, Zangen A (2007) Repeated electrical stimulation of reward-related brain regions affects cocaine but not "natural" reinforcement. J Neurosci 27:14179-14189.

Maldonado-Irizarry CS, Kelley AE (1994) Differential behavioral effects following microinjection of an NMDA antagonist into nucleus accumbens subregions. Psychopharmacology (Berl) 116:65-72.

Marowsky A, Yanagawa Y, Obata K, Vogt KE (2005) A specialized subclass of interneurons mediates dopaminergic facilitation of amygdala function. Neuron 48:1025-1037.

Martin JH, Ghez C (1999) Pharmacological inactivation in the analysis of the central control of movement. J Neurosci Methods 86:145-159.

Martin M, Chen BT, Hopf FW, Bowers MS, Bonci A (2006) Cocaine selfadministration selectively abolishes LTD in the core of the nucleus accumbens. Nat Neurosci 9:868-869.

McFarland K, Kalivas PW (2001) The circuitry mediating cocaine-induced reinstatement of drug-seeking behavior. J Neurosci 21:8655-8663.

McFarland K, Lapish CC, Kalivas PW (2003) Prefrontal glutamate release into the core of the nucleus accumbens mediates cocaine-induced reinstatement of drug-seeking behavior. J Neurosci 23:3531-3537.

McFarland K, Davidge SB, Lapish CC, Kalivas PW (2004) Limbic and motor circuitry underlying footshock-induced reinstatement of cocaine seeking behavior. J Neurosci 24:1551-1560.

McLaughlin J, See RE (2003) Selective inactivation of the dorsomedial prefrontal cortex and the basolateral amygdala attenuates conditioned-cued reinstatement of extinguished cocaine seeking behavior in rats. Psychopharmacology (Berl) 168:57-65.

Ongür D, Price JL (2000) The organization of networks within the orbital and medial prefrontal cortex of rats, monkeys and humans. Cereb Cortex 10:206-219.

Paxinos G, Watson C (1986) The rat brain in stereotaxic coordinates. New York: Academic.

Quirk GJ, Likhtik E, Pelletier JG, Paré D (2003) Stimulation of medial prefrontal cortex decreases the responsiveness of central amygdala output neurons. J Neurosci 23:8800-8807.

Quirk GJ, Garcia R, González-Lima F (2006) Prefrontal mechanisms in extinction of conditioned fear. Biol Psychiatry 60:337-343.

Reynolds SM, Berridge RC (2003) Glutamate motivational ensembles in the nucleus accumbens: rostrocaudal shell gradients of fear and feeding. Eur J Neurosci 17:2187-2200.

Rizvi TA, Ennis M, Behbehani MM, Shipley MT (1991) Connections between the central nucleus of the amygdala and the midbrain periaqueductal gray: topography and reciprocity. J Comp Neurol 303:121-131.

Schmidt HD, Anderson SM, Pierce RC (2006) Stimulation of D1-like or D2 dopamine receptors in the shell, but not the core, of the nucleus accumbens reinstates cocaine-seeking behaviour in the rat. Eur J Neurosci 23:219-228.

Sesack SR, Deutch AY, Roth RH, Bunney BS (1989) Topographical organization of the efferent projections of the medial prefrontal cortex in the rat: an anterograde tract-tracing study with Phaseolus vulgaris leucoagglutinin. J Comp Neurol 290:213-242.

Sutton MA, Schmidt EF, Choi KH, Schad CA, Whisler K, Simmons D, Karanian DA, Monteggia LM, Neve RL, Self DW (2003) Extinction-induced upregulation in AMPA receptors reduces cocaine seeking behaviour. Nature 421:70-75.

van Duuren E, van der Plasse G, van der Blom R, Joosten RN, Mulder AB, Pennartz CM, Feenstra MG (2007) Pharmacological manipulation of neuronal ensemble activity by reverse microdialysis in freely moving rats: a comparative study of the effects of tetrodotoxin, lidocaine, and muscimol. J Pharmacol Exp Ther 323:61-69.

Vidal-Gonzalez I, Vidal-Gonzalez B, Rauch SL, Quirk GJ (2006) Microstimulation reveals opposing influences of prelimbic and infralimbic cortex on the expression of conditioned fear. Learn Mem 13:728-733.

Voorn P, Vanderschuren LJ, Groenewegen HJ, Robbins TW, Pennartz CM (2004) Putting a spin on the dorsal-ventral divide of the striatum. Trends Neurosci 27:468-474. 